\title{
Bregman $f$-Projection Operator with Applications to Variational Inequalities in Banach Spaces
}

\author{
Chin-Tzong Pang, ${ }^{1}$ Eskandar Naraghirad, ${ }^{2}$ and Ching-Feng Wen ${ }^{3}$ \\ ${ }^{1}$ Department of Information Management, Yuan Ze University, Chung-Li 32003, Taiwan \\ ${ }^{2}$ Department of Mathematics, Yasouj University, Yasouj 75918, Iran \\ ${ }^{3}$ Center for Fundamental Science, Kaohsiung Medical University, Kaohsiung 807, Taiwan
}

Correspondence should be addressed to Eskandar Naraghirad; eskandarrad@gmail.com

Received 24 January 2014; Accepted 5 February 2014; Published 20 March 2014

Academic Editor: Jen-Chih Yao

Copyright (C) 2014 Chin-Tzong Pang et al. This is an open access article distributed under the Creative Commons Attribution License, which permits unrestricted use, distribution, and reproduction in any medium, provided the original work is properly cited.

Using Bregman functions, we introduce the new concept of Bregman generalized $f$-projection operator Proj ${ }_{C}^{f, g}: E^{*} \rightarrow C$, where $E$ is a reflexive Banach space with dual space $E^{*} ; f: E \rightarrow \mathbb{R} \cup\{+\infty\}$ is a proper, convex, lower semicontinuous and bounded from below function; $g: E \rightarrow \mathbb{R}$ is a strictly convex and Gâteaux differentiable function; and $C$ is a nonempty, closed, and convex subset of $E$. The existence of a solution for a class of variational inequalities in Banach spaces is presented.

\section{Introduction}

Many nonlinear problems in functional analysis can be reduced to the search of fixed points of nonlinear operators. See, for example, [1-14] and the references therein. Let $E$ be a (real) Banach space with norm $\|\cdot\|$ and dual space $E^{*}$. For any $x$ in $E$, we denote the value of $x^{*}$ in $E^{*}$ at $x$ by $\left\langle x, x^{*}\right\rangle$. When $\left\{x_{n}\right\}_{n \in \mathbb{N}}$ is a sequence in $E$, we denote the strong convergence of $\left\{x_{n}\right\}_{n \in \mathbb{N}}$ to $x \in E$ by $x_{n} \rightarrow x$ and the weak convergence by $x_{n} \rightarrow x$. Let $C$ be a nonempty subset of $E$ and $T: C \rightarrow E$ be a mapping. We denote by $F(T)=\{x \in C: T x=x\}$ the set of fixed points of $T$. Let $C$ be a nonempty, closed, and convex subset of a smooth Banach space $E$; let $T$ be a mapping from $C$ into itself. A point $p \in C$ is said to be an asymptotic fixed point [15] of $T$ if there exists a sequence $\left\{x_{n}\right\}_{n \in \mathbb{N}}$ in $C$ which converges weakly to $p$ and $\lim _{n \rightarrow \infty}\left\|x_{n}-T x_{n}\right\|=0$. We denote the set of all asymptotic fixed points of $T$ by $\widehat{F}(T)$. A point $p \in C$ is called a strong asymptotic fixed point of $T$ if there exists a sequence $\left\{x_{n}\right\}_{n \in \mathbb{N}}$ in $C$ which converges strongly to $p$ and $\lim _{n \rightarrow \infty}\left\|x_{n}-T x_{n}\right\|=0$. We denote the set of all strong asymptotic fixed points of $T$ by $\widetilde{F}(T)$.

We recall the definition of Bregman distances. Let $g$ : $E \rightarrow \mathbb{R}$ be a strictly convex and Gâteaux differentiable function on a Banach space $E$. The Bregman distance [16] (see also $[17,18]$ ) corresponding to $g$ is the function $D_{g}$ : $E \times E \rightarrow \mathbb{R}$ defined by

$$
D_{g}(x, y)=g(x)-g(y)-\langle x-y, \nabla g(y)\rangle, \forall x, y \in E .
$$

It follows from the strict convexity of $g$ that $D_{g}(x, y) \geq 0$ for all $x, y$ in $E$. However, $D_{g}$ might not be symmetric and $D_{g}$ might not satisfy the triangular inequality.

When $E$ is a smooth Banach space, setting $g(x)=\|x\|^{2}$ for all $x$ in $E$, we have that $\nabla g(x)=2 J x$ for all $x$ in $E$. Here $J$ is the normalized duality mapping from $E$ into $E^{*}$. Hence, $D_{g}(\cdot, \cdot)$ reduces to the usual map $\phi(\cdot, \cdot)$ as

$$
D_{g}(x, y)=\phi(x, y):=\|x\|^{2}-2\langle x, J y\rangle+\|y\|^{2}, \quad \forall x, y \in E .
$$

If $E$ is a Hilbert space, then $D_{g}(x, y)=\|x-y\|^{2}$.

Let $g: E \rightarrow \mathbb{R}$ be strictly convex and Gâteaux differentiable and $C \subseteq E$ be nonempty. A mapping $T: C \rightarrow$ $E$ is said to be

(i) Bregman nonexpansive if

$$
D_{g}(T x, T y) \leq D_{g}(x, y), \quad \forall x, y \in C .
$$


(ii) Bregman quasi-nonexpansive if $F(T) \neq \emptyset$ and

$$
D_{g}(p, T x) \leq D_{g}(p, x), \quad \forall x \in C, \forall p \in F(T) .
$$

(iii) Bregman relatively nonexpansive if the following conditions are satisfied:

(1) $F(T)$ is nonempty;

(2) $D_{g}(p, T v) \leq D_{g}(p, v), \forall p \in F(T), v \in C$;

(3) $\widehat{F}(T)=F(T)$;

(iv) Bregman weak relatively nonexpansive if the following conditions are satisfied:

(1) $F(T)$ is nonempty;

(2) $D_{g}(p, T v) \leq D_{g}(p, v), \forall p \in F(T), v \in C$;

(3) $\widetilde{F}(T)=F(T)$.

It is clear that any Bregman relatively nonexpansive mapping is a Bregman quasi-nonexpansive mapping. It is also obvious that every Bregman relatively nonexpansive mapping is a Bregman weak relatively nonexpansive mapping, but the converse is not true in general; see, for example, [19]. Indeed, for any mapping $T: C \rightarrow C$ we have $F(T) \subset \widetilde{F}(T) \subset \widehat{F}(T)$. If $T$ is Bregman relatively nonexpansive, then $F(T)=\widetilde{F}(T)=$ $\widehat{F}(T)$.

Let $E$ be a reflexive Banach space, let $f: E \rightarrow \mathbb{R} \cup\{+\infty\}$ be a proper, convex, lower semicontinuous function, let $g$ : $E \rightarrow \mathbb{R}$ be strictly convex and Gâteaux differentiable, and let $C \subseteq E$ be nonempty. We define a functional $H: E \times E^{*} \rightarrow$ $\mathbb{R} \cup\{+\infty\}$ by

$$
\begin{array}{r}
H\left(x, x^{*}\right)=g(x)-\left\langle x, x^{*}\right\rangle+g^{*}\left(x^{*}\right)+f(x), \\
x \in E, \quad x^{*} \in E^{*} .
\end{array}
$$

It could easily be seen that $H$ satisfies the following properties:

(1) $H\left(x, x^{*}\right)$ is convex and continuous with respect to $x^{*}$ when $x$ is fixed;

(2) $H\left(x, x^{*}\right)$ is convex and lower semicontinuous with respect to $x$ when $x^{*}$ is fixed.

Definition 1. Let $E$ be a Banach space with dual space $E^{*}$, let $f: E \rightarrow \mathbb{R} \cup\{+\infty\}$ be a proper, convex, lower semicontinuous function, let $g: E \rightarrow \mathbb{R}$ be strictly convex and Gâteaux differentiable, and let $C$ be a nonempty, closed subset of $E$. We say that $\operatorname{Proj}_{C}^{f, g}: E^{*} \rightarrow 2^{C}$ is a Bregman generalized $f$-projection operator if

$$
\operatorname{Proj}_{C}^{f, g}=\left\{z \in C: H\left(z, x^{*}\right)=\inf _{y \in C} H\left(y, x^{*}\right)\right\}, \quad \forall x^{*} \in E^{*} .
$$

In this paper, using Bregman functions, we introduce the new concept of Bregman generalized $f$-projection operator $\operatorname{Proj}_{C}^{f, g}: E^{*} \rightarrow C$, where $E$ is a reflexive Banach space with dual space $E^{*}, f: E \rightarrow \mathbb{R} \cup\{+\infty\}$ is a proper, convex, lower semicontinuous, and bounded from below function, $g: E \rightarrow$ $\mathbb{R}$ is a strictly convex and Gâteaux differentiable function, and $C$ is a nonempty, closed, and convex subset of $E$. The existence of a solution for a class of variational inequalities in Banach spaces is presented. Our results improve and generalize some known results in the current literature; see, for example, [20, 21].

\section{Properties of Bregman Functions and Bregman Distances}

Let $E$ be a (real) Banach space, and let $g: E \rightarrow \mathbb{R}$. For any $x$ in $E$, the gradient $\nabla g(x)$ is defined to be the linear functional in $E^{*}$ such that

$$
\langle y, \nabla g(x)\rangle=\lim _{t \rightarrow 0} \frac{g(x+t y)-g(x)}{t}, \quad \forall y \in E .
$$

The function $g$ is said to be Gâteaux differentiable at $x$ if $\nabla g(x)$ is well defined, and $g$ is Gâteaux differentiable if it is Gâteaux differentiable everywhere on $E$. We call $g$ Fréchet differentiable at $x$ (see, for example, [22, page 13] or [23, page 508]) if, for all $\epsilon>0$, there exists $\delta>0$ such that

$$
\begin{aligned}
& |g(y)-g(x)-\langle y-x, \nabla g(x)\rangle| \\
& \quad \leq \epsilon\|y-x\| \quad \text { whenever }\|y-x\| \leq \delta .
\end{aligned}
$$

The function $g$ is said to be Fréchet differentiable if it is Fréchet differentiable everywhere.

For any $r>0$, let $B_{r}:=\{z \in E:\|z\| \leq r\}$. A function $g: E \rightarrow \mathbb{R}$ is said to be

(i) strongly coercive if

$$
\lim _{\left\|x_{n}\right\| \rightarrow+\infty} \frac{g\left(x_{n}\right)}{\left\|x_{n}\right\|}=+\infty
$$

(ii) locally bounded if $g\left(B_{r}\right)$ is bounded for all $r>0$;

(iii) locally uniformly smooth on $E$ ([24, pages 207, 221]) if the function $\sigma_{r}:[0,+\infty) \rightarrow[0,+\infty]$, defined by

$$
\begin{aligned}
\sigma_{r}(t)=\sup _{x \in B_{r}, y \in S_{E}, \alpha \in(0,1)}( & (\alpha g(x+(1-\alpha) t y) \\
& +(1-\alpha) g(x-\alpha t y)-g(x)) \\
& \left.\times(\alpha(1-\alpha))^{-1}\right),
\end{aligned}
$$

satisfies

$$
\lim _{t \downarrow 0} \frac{\sigma_{r}(t)}{t}=0, \quad \forall r>0
$$

(iv) locally uniformly convex on $E$ (or uniformly convex on bounded subsets of $E$ ([24, pages 203, 221])) if the 
gauge $\rho_{r}:[0,+\infty) \rightarrow[0,+\infty]$ of uniform convexity of $g$, defined by

$$
\begin{aligned}
\rho_{r}(t)=\inf _{x, y \in B_{r},\|x-y\|=t, \alpha \in(0,1)}((\alpha g(x)+(1-\alpha) g(y) \\
-g(\alpha x+(1-\alpha) y)) \\
\left.\times(\alpha(1-\alpha))^{-1}\right),
\end{aligned}
$$

satisfies

$$
\rho_{r}(t)>0, \quad \forall r, t>0
$$

For a locally uniformly convex map $g: E \rightarrow \mathbb{R}$, we have

$$
\begin{aligned}
g(\alpha x+(1-\alpha) y) \leq & \alpha g(x)+(1-\alpha) g(y) \\
& -\alpha(1-\alpha) \rho_{r}(\|x-y\|),
\end{aligned}
$$

for all $x, y$ in $B_{r}$ and for all $\alpha$ in $(0,1)$.

Let $E$ be a Banach space and $g: E \rightarrow \mathbb{R}$ a strictly convex and Gâteaux differentiable function. By (1), the Bregman distance satisfies [16]

$$
\begin{aligned}
D_{g}(x, z)= & D_{g}(x, y)+D_{g}(y, z) \\
& +\langle x-y, \nabla g(y)-\nabla g(z)\rangle, \quad \forall x, y, z \in E .
\end{aligned}
$$

In particular,

$$
\begin{array}{r}
D_{g}(x, y)=-D_{g}(y, x)+\langle y-x, \nabla g(y)-\nabla g(x)\rangle \\
\forall x, y \in E .
\end{array}
$$

We call a function $g: E \rightarrow(-\infty,+\infty]$ lower semicontinuous if $\{x \in E: g(x) \leq r\}$ is closed for all $r$ in $\mathbb{R}$. For a lower semicontinuous convex function $g: E \rightarrow \mathbb{R}$, the subdifferential $\partial g$ of $g$ is defined by

$$
\partial g(x)=\left\{x^{*} \in E^{*}: g(x)+\left\langle y-x, x^{*}\right\rangle \leq g(y), \quad \forall y \in E\right\}
$$

for all $x$ in $E$. It is well known that $\partial g \subset E \times E^{*}$ is maximal monotone $[25,26]$. For any lower semicontinuous convex function $g: E \rightarrow(-\infty,+\infty]$, the conjugate function $g^{*}$ of $g$ is defined by

$$
g^{*}\left(x^{*}\right)=\sup _{x \in E}\left\{\left\langle x, x^{*}\right\rangle-g(x)\right\}, \quad \forall x^{*} \in E^{*} .
$$

It is well known that

$$
\begin{array}{cl}
g(x)+g^{*}\left(x^{*}\right) \geq\left\langle x, x^{*}\right\rangle, & \forall\left(x, x^{*}\right) \in E \times E^{*}, \\
\left(x, x^{*}\right) \in \partial g \text { is equivalent to } & g(x)+g^{*}\left(x^{*}\right)=\left\langle x, x^{*}\right\rangle .
\end{array}
$$

We also know that if $g: E \rightarrow(-\infty,+\infty]$ is a proper lower semicontinuous convex function, then $g^{*}: E^{*} \rightarrow$ $(-\infty,+\infty]$ is a proper weak ${ }^{*}$ lower semicontinuous convex function. Here, saying $g$ is proper we mean that dom $g:=$ $\{x \in E: g(x)<+\infty\} \neq \emptyset$.

The following definition is slightly different from that in Butnariu and Iusem [22].
Definition 2 (see [23]). Let $E$ be a Banach space. A function $g: E \rightarrow \mathbb{R}$ is said to be a Bregman function if the following conditions are satisfied:

(1) $g$ is continuous, strictly convex, and Gâteaux differentiable;

(2) the set $\left\{y \in E: D_{g}(x, y) \leq r\right\}$ is bounded for all $x$ in $E$ and $r>0$.

The following lemma follows from Butnariu and Iusem [22] and Zălinescu [24].

Lemma 3. Let $E$ be a reflexive Banach space and $g: E \rightarrow \mathbb{R}$ a strongly coercive Bregman function. Then

(1) $\nabla g: E \rightarrow E^{*}$ is one-to-one, onto, and norm-to-weak ${ }^{*}$ continuous;

(2) $\langle x-y, \nabla g(x)-\nabla g(y)\rangle=0$ if and only if $x=y$;

(3) $\left\{x \in E: D_{g}(x, y) \leq r\right\}$ is bounded for all $y$ in $E$ and $r>0$

(4) dom $g^{*}=E^{*}, g^{*}$ is Gâteaux differentiable and $\nabla g^{*}=$ $(\nabla g)^{-1}$. 3.6.4].

The following two results follow from [24, Proposition

Proposition 4. Let $E$ be a reflexive Banach space and let $g$ : $E \rightarrow \mathbb{R}$ be a convex function which is locally bounded. The following assertions are equivalent:

(1) $g$ is strongly coercive and locally uniformly convex on E;

(2) dom $g^{*}=E^{*}, g^{*}$ is locally bounded and locally uniformly smooth on $E$;

(3) dom $g^{*}=E^{*}, g^{*}$ is Fréchet differentiable and $\nabla g^{*}$ is uniformly norm-to-norm continuous on bounded subsets of $E^{*}$.

Proposition 5. Let $E$ be a reflexive Banach space and $g: E \rightarrow$ $\mathbb{R}$ a continuous convex function which is strongly coercive. The following assertions are equivalent:

(1) $g$ is locally bounded and locally uniformly smooth on E;

(2) $g^{*}$ is Fréchet differentiable and $\nabla g^{*}$ is uniformly normto-norm continuous on bounded subsets of $E$;

(3) dom $g^{*}=E^{*}, g^{*}$ is strongly coercive and locally uniformly convex on $E$.

Let $E$ be a Banach space and let $C$ be a nonempty convex subset of $E$. Let $g: E \rightarrow \mathbb{R}$ be a strictly convex and Gâteaux differentiable function. Then, we know from [27] that for $x$ in $E$ and $x_{0}$ in $C$, we have

$$
D_{g}\left(x_{0}, x\right)=\min _{y \in C} D_{g}(y, x)
$$

iff $\left\langle y-x_{0}, \nabla g(x)-\nabla g\left(x_{0}\right)\right\rangle \leq 0, \forall y \in C$. 
Further, if $C$ is a nonempty, closed, and convex subset of a reflexive Banach space $E$ and $g: E \rightarrow \mathbb{R}$ is a strongly coercive Bregman function, then, for each $x$ in $E$, there exists a unique $x_{0}$ in $C$ such that

$$
D_{g}\left(x_{0}, x\right)=\min _{y \in C} D_{g}(y, x)
$$

The Bregman projection $\operatorname{proj}_{C}^{g}$ from $E$ onto $C$ defined by $\operatorname{proj}_{C}^{g}(x)=x_{0}$ has the following property:

$$
\begin{array}{r}
D_{g}\left(y, \operatorname{proj}_{C}^{g} x\right)+D_{g}\left(\operatorname{proj}_{C}^{g} x, x\right) \leq D_{g}(y, x), \\
\forall y \in C, \quad \forall x \in E .
\end{array}
$$

See [22] for details.

Lemma 6 (see [9]). Let $E$ be a Banach space and $g: E \rightarrow$ $\mathbb{R}$ a Gâteaux differentiable function which is locally uniformly convex on $E$. Let $\left\{x_{n}\right\}_{n \in \mathbb{N}}$ and $\left\{y_{n}\right\}_{n \in \mathbb{N}}$ be bounded sequences in $E$. Then the following assertions are equivalent:

(1) $\lim _{n \rightarrow \infty} D_{g}\left(x_{n}, y_{n}\right)=0$;

(2) $\lim _{n \rightarrow \infty}\left\|x_{n}-y_{n}\right\|=0$.

Lemma 7 (see $[23,28])$. Let $E$ be a reflexive Banach space, let $g: E \rightarrow \mathbb{R}$ be a strongly coercive Bregman function, and let $V$ be the function defined by

$$
V\left(x, x^{*}\right)=g(x)-\left\langle x, x^{*}\right\rangle+g^{*}\left(x^{*}\right), \quad \forall x \in E, \forall x^{*} \in E^{*} .
$$

The following assertions hold:

(1) $D_{g}\left(x, \nabla g^{*}\left(x^{*}\right)\right)=V\left(x, x^{*}\right)$ for all $x$ in $E$ and $x^{*}$ in $E^{*}$;

(2) $V\left(x, x^{*}\right)+\left\langle\nabla g^{*}\left(x^{*}\right)-x, y^{*}\right\rangle \leq V\left(x, x^{*}+y^{*}\right)$ for all $x$ in $E$ and $x^{*}, y^{*}$ in $E^{*}$.

It also follows from the definition that $V$ is convex in the second variable $x^{*}$, and

$$
V(x, \nabla g(y))=D_{g}(x, y) .
$$

Lemma 8 (see [29, Proposition 23.1]). Let E be a real Banach space and let $f: E \rightarrow \mathbb{R} \cup\{+\infty\}$ be a lower semicontinuous convex function. Then there exist $x^{*} \in E^{*}$ and $a \in \mathbb{R}$ such that

$$
f(x) \geq x^{*}(x)+a, \quad \forall x \in E .
$$

\section{Properties of Bregman $f$-Projection Operator Proj ${ }_{C}^{f, g}$}

Theorem 9. Let $C$ be a nonempty, closed, and convex subset of a reflexive Banach space $E$. Let $f: E \rightarrow \mathbb{R} \cup\{+\infty\}$ be a proper, convex, lower semicontinuous function and let $g: E \rightarrow \mathbb{R}$ be strictly convex, continuous, strongly coercive, Gâteaux differentiable, locally bounded, and locally uniformly convex on $E$. Then $\operatorname{Proj}_{C}^{f, g}\left(x^{*}\right) \neq \emptyset$ for all $x^{*} \in E^{*}$.
Proof. Let $x^{*} \in E^{*}$ and $\lambda=\inf _{y \in C} H\left(y, x^{*}\right)$. Then there exists a sequence $\left\{x_{n}\right\}_{n \in \mathbb{N}} \subset C$ such that $\lambda=\lim _{n \rightarrow \infty} H\left(x_{n}, x^{*}\right)$. We consider the following two possible cases.

Case 1. If $C$ is bounded, then there exists a subsequence $\left\{x_{n_{j}}\right\}_{j \in \mathbb{N}}$ of $\left\{x_{n}\right\}_{n \in \mathbb{N}}$ and $x \in C$ such that $x_{n_{j}} \rightarrow x$ as $j \rightarrow$ $\infty$. Since $H\left(z, x^{*}\right)$ is convex and lower semicontinuous with respect to $z$, we deduce that $H\left(z, x^{*}\right)$ is convex and weakly lower semicontinuous with respect to $z$. This implies that

$$
\begin{aligned}
H\left(x, x^{*}\right) & \leq \liminf _{n \rightarrow \infty} H\left(x_{n}, x^{*}\right)=\lim _{n \rightarrow \infty} H\left(x_{n}, x^{*}\right) \\
& =\inf _{y \in C} H\left(x_{n}, x^{*}\right)
\end{aligned}
$$

and hence $x \in \operatorname{Proj}_{C}^{f, g}\left(x^{*}\right)$. This shows that $\operatorname{Proj}_{C}^{f, g} \neq \emptyset$.

Case 2. Assume that $C$ is unbounded. Since $f: C \rightarrow \mathbb{R} \cup$ $\{+\infty\}$ is proper, convex, and lower semicontinuous, we know that the function $f_{C}: E \rightarrow \mathbb{R} \cup\{+\infty\}$, defined by

$$
f_{C}(x)= \begin{cases}f(x), & \text { if } x \in C, \\ +\infty, & \text { if } x \notin C\end{cases}
$$

is proper, convex, and lower semicontinuous. In view of Lemma 8, there exist $x^{*} \in E^{*}$ and $a \in \mathbb{R}$ such that

$$
f_{C}(x) \geq\left\langle x, x^{*}\right\rangle+a, \quad \forall x \in E .
$$

This implies that for any $x^{*} \in E^{*}$ and $x \in C$

$$
\begin{aligned}
H\left(x, x^{*}\right) & =g(x)-\left\langle x, x^{*}\right\rangle+g^{*}\left(x^{*}\right)+f(x) \\
& \geq g(x)+g^{*}\left(x^{*}\right)+a .
\end{aligned}
$$

Next, we show that $\left\{x_{n}\right\}_{n \in \mathbb{N}}$ is bounded. If not, then there exists a subsequence $\left\{x_{n_{j}}\right\}_{j \in \mathbb{N}}$ of $\left\{x_{n}\right\}_{n \in \mathbb{N}}$ such that $\left\|x_{n_{k}}\right\| \rightarrow$ $+\infty$ as $k \rightarrow \infty$. Since $g$ is strongly coercive, we conclude that

$$
\lim _{\left\|x_{n_{k}}\right\| \rightarrow+\infty} \frac{H\left(x_{n_{k}}, x^{*}\right)}{\left\|x_{n_{k}}\right\|} \geq \lim _{\left\|x_{n_{k}}\right\| \rightarrow+\infty} \frac{g\left(x_{n_{k}}\right)}{\left\|x_{n_{k}}\right\|}=+\infty .
$$

This implies that

$$
\lim _{\left\|x_{n_{k}}\right\| \rightarrow+\infty} H\left(x_{n_{k}}, x^{*}\right)=+\infty
$$

Since $f$ is proper in $C$, we obtain that $\lambda=\inf _{y \in C} H\left(y, x^{*}\right)=$ $\lim _{n \rightarrow \infty} H\left(x_{n}, x^{*}\right)<+\infty$ which contradicts (31). By a similar argument, as in Case 1 , we can prove that $\operatorname{Proj}_{C}^{f, g}\left(x^{*}\right) \neq \emptyset$ which completes the proof.

Theorem 10. Let $C$ be a nonempty, closed, and convex subset of a reflexive Banach space $E$. Let $g: E \rightarrow \mathbb{R}$ be strictly convex, continuous, strongly coercive, Gâteaux differentiable, locally bounded, and locally uniformly convex on E. Then the following assertions hold:

(i) for any given $x^{*} \in E^{*}, \operatorname{Proj}_{C}^{f, g}\left(x^{*}\right)$ is a nonempty, closed, and convex subset of $C$; 
(ii) $\operatorname{Proj}_{C}^{f, g}$ is monotone; that is, for any $x^{*}, y^{*} \in E^{*}, x \in$ $\operatorname{Proj}_{C}^{f, g}\left(x^{*}\right)$ and $y \in \operatorname{Proj}_{C}^{f, g}\left(y^{*}\right)$,

$$
\left\langle x-y, x^{*}-y^{*}\right\rangle \geq 0
$$

(iii) For any given $x^{*} \in E^{*}, x \in \operatorname{Proj}_{C}^{f, g}\left(x^{*}\right)$ if and only if

$$
\left\langle x-y, x^{*}-\nabla g(x)\right\rangle+f(y)-f(x) \geq 0
$$

Proof. (i) Let $x^{*} \in E^{*}$ be fixed. In view of Theorem 9, we conclude that $\operatorname{Proj}_{C}^{f, g}\left(x^{*}\right) \neq \emptyset$. According to (20) we have $g(x)+g^{*}\left(x^{*}\right)-\left\langle x, x^{*}\right\rangle \geq 0, \forall\left(x, x^{*}\right) \in E \times E^{*}$. Let us prove that $\operatorname{Proj}_{C}^{f, g}\left(x^{*}\right)$ is closed. Let $\left\{x_{n}\right\}_{n \in \mathbb{N}} \subset \operatorname{Proj}_{C}^{f, g}\left(x^{*}\right)$ and $x_{n} \rightarrow x$ as $n \rightarrow \infty$. In view of (6), we deduce that

$$
\begin{aligned}
G\left(x, x^{*}\right) & \leq \liminf _{n \rightarrow \infty} H\left(x_{n}, x^{*}\right) \\
& =\liminf _{n \rightarrow \infty} H\left(x_{n}, x^{*}\right)=\inf _{y \in C} H\left(y, x^{*}\right) .
\end{aligned}
$$

This implies that $x \in \operatorname{Proj}_{C}^{f, g}\left(x^{*}\right)$ and hence $\operatorname{Proj}_{C}^{f, g}\left(x^{*}\right)$ is closed. Next, we show that $\operatorname{Proj}_{C}^{f, g}\left(x^{*}\right)$ is convex. Let $x_{1}, x_{2} \in$ $\operatorname{Proj}_{C}^{f, g}\left(x^{*}\right)$ and $0 \leq t \leq 1$. By the property (2) of the functional $H$, we obtain

$$
\begin{aligned}
H & \left(t x_{1}+(1-t) x_{2}, x^{*}\right) \\
& \leq t H\left(x_{1}, x^{*}\right)+(1-t) H\left(x_{2}, x^{*}\right) \\
& =t \inf _{y \in C} H\left(y, x^{*}\right)+(1-t) \inf _{y \in C} H\left(y, x^{*}\right) \\
& =\inf _{y \in C} H\left(y, x^{*}\right) .
\end{aligned}
$$

Thus, we have $t x_{1}+(1-t) x_{2} \in \operatorname{Proj}_{C}^{f, g}\left(x^{*}\right)$ and hence $\operatorname{Proj}_{C}^{f, g}\left(x^{*}\right)$ is convex.

(ii) Let $x_{1}^{*}, x_{2}^{*} \in E^{*}, x_{1} \in \operatorname{Proj}_{C}^{f, g}\left(x_{1}^{*}\right)$, and $x_{2} \in$ $\operatorname{Proj}_{C}^{f, g}\left(x_{2}^{*}\right)$. Then we have

$$
\begin{aligned}
& g\left(x_{1}\right)-\left\langle x_{1}, x_{1}^{*}\right\rangle+g^{*}\left(x_{1}^{*}\right)+f\left(x_{1}\right) \\
& \quad \leq g\left(x_{2}\right)-\left\langle x_{2}, x_{2}^{*}\right\rangle+g^{*}\left(x_{2}^{*}\right)+f\left(x_{2}\right), \\
& g\left(x_{2}\right)-\left\langle x_{2}, x_{2}^{*}\right\rangle+g^{*}\left(x_{2}^{*}\right)+f\left(x_{2}\right) \\
& \quad \leq g\left(x_{1}\right)-\left\langle x_{1}, x_{1}^{*}\right\rangle+g^{*}\left(x_{1}^{*}\right)+f\left(x_{1}\right) .
\end{aligned}
$$

In view of (37), we conclude that $\operatorname{Proj}_{C}^{f, g}\left(x^{*}\right)$ is monotone. that

(iii) It is a simple matter to see that $x \in \operatorname{Proj}_{C}^{f, g}\left(x^{*}\right)$ implies

$$
\left\langle x^{*}-\nabla g(x), x-y\right\rangle+f(y)-f(x) \geq 0, \quad \forall y \in C .
$$

To this end, let $y \in C$ and $t \in(0,1]$ be arbitrarily chosen. By the definition of $\operatorname{Proj}_{C}^{f, g}\left(x^{*}\right)$ we see that

$$
H\left(x, x^{*}\right) \leq H\left(x+t(y-x), x^{*}\right) .
$$

Therefore,

$$
\begin{aligned}
g(x) & +g^{*}\left(x^{*}\right)-\left\langle x, x^{*}\right\rangle+f(x) \\
\leq & g(x+t(y-x))+g^{*}\left(x^{*}\right) \\
& -\left\langle x+t(y-x), x^{*}\right\rangle+f(x+t(y-x)) \\
\leq & g(x+t(y-x))+g^{*}\left(x^{*}\right) \\
& -\left\langle x+t(y-x), x^{*}\right\rangle+t f(y)+(1-t) f(x)
\end{aligned}
$$

and hence

$$
\left\langle t(y-x), x^{*}\right\rangle \leq g(x+t(y-x))+t(f(y)-f(x)) .
$$

On the other hand, by the definition of Bregman distance, we obtain that

$$
g(x)+g(x+t(y-x)) \geq\langle t(x-y), \nabla g(x+t(y-x))\rangle .
$$

This, together with (41), implies that

$$
\langle x-y, \nabla g(x+t(y-x))\rangle \geq f(x)-f(y)+\left\langle x-y, x^{*}\right\rangle .
$$

Since $\nabla g$ is demi-continuous, letting $t \rightarrow 0$ in (43), we conclude that

$$
\left\langle x-y, \nabla g(x)-x^{*}\right\rangle+f(y)-f(x) \geq 0 .
$$

Conversely, assume that

$$
\left\langle x-y, \nabla g(x)-x^{*}\right\rangle+f(y)-f(x) \geq 0, \quad \forall y \in K .
$$

This implies that

$$
\begin{aligned}
& g(y)-g(x) \geq\langle x-y, \nabla g(x)\rangle \\
& \geq\left\langle x-y, x^{*}\right\rangle+f(y)-f(x) \geq 0 \\
& \forall y \in K .
\end{aligned}
$$

\section{Applications to Variational Inequalities}

In this section, we investigate the existence of solution to the following variational inequality problem: find the point $x \in C$ such that

$$
\langle y-x, A x\rangle+f(y)-f(x) \geq 0, \quad \forall y \in C,
$$

where $C$ is a nonempty, closed, and convex subset of the Banach space $E$, and $A: C \rightarrow E^{*}$ and $f: C \rightarrow \mathbb{R} \cup\{+\infty\}$ are two mappings.

Definition 11 (KKM mapping [30]). Let $C$ be a nonempty subset of a linear space $X$. A set-valued mapping $G: C \rightarrow 2^{X}$ is 
called a KKM mapping if, for any finite subset $\left\{y_{1}, y_{2}, \ldots, y_{n}\right\}$ of $C$, we have

$$
\operatorname{co}\left\{y_{1}, y_{2}, \ldots, y_{n}\right\} \subset \bigcup_{i=1}^{n} G\left(y_{i}\right)
$$

where $\operatorname{co}\left\{y_{1}, y_{2}, \ldots, y_{n}\right\}$ denotes the convex hull of $\left\{y_{1}, y_{2}, \ldots, y_{n}\right\}$.

Lemma 12 (Fan KKM Theorem [30]). Let $C$ be a nonempty convex subset of a Hausdorff topological vector $X$ and let $G$ : $C \rightarrow 2^{X}$ be a KKM mapping with closed values. If there exists a point $y_{0} \in C$ such that $G\left(y_{0}\right)$ is a compact subset of $C$, then $\bigcap_{y \in C} G(y) \neq \emptyset$.

Theorem 13. Let $C$ be a nonempty, closed, and convex subset of a reflexive Banach space $E$ with dual space $E^{*}$. Let $g: E \rightarrow$ $\mathbb{R}$ be strictly convex, continuous, strongly coercive, Gâteaux differentiable, locally bounded and locally uniformly convex on E. Let $A: C \rightarrow E^{*}$ be a continuous mapping and $f: E \rightarrow$ $\mathbb{R} \cup\{+\infty\}$ be a proper, convex, lower semicontinuous function. If there exists an element $y_{0} \in C$ such that

$$
\begin{aligned}
& \left\{x \in C:\left\langle y_{0}-x, \nabla g(x)-A x\right\rangle\right. \\
& \left.\quad+g(x)+f(x) \leq g\left(y_{0}\right)+f\left(y_{0}\right)\right\}
\end{aligned}
$$

is a compact subset of $C$, then the variational inequality (47) has a solution.

Proof. In view of Theorem 10, we need to prove that the following inclusion has a solution:

$$
x \in \operatorname{Proj}_{C}^{f, g}(\nabla g(x)-A x) .
$$

We define a set-valued mapping $V: C \rightarrow 2^{C}$ by

$$
\begin{aligned}
& V(y) \\
& \quad=\{x \in C: H(x, \nabla g(x)-A x) \leq H(y, \nabla g(x)-A x)\} .
\end{aligned}
$$

It is obvious that, for any $y \in C, V(y) \neq \emptyset$. Let us prove that $V(y)$ is closed for any $y \in C$. Let $\left\{x_{n}\right\}_{n \in \mathbb{N}} \subset V(y)$ and $x_{n} \rightarrow x$ as $n \rightarrow \infty$. Then,

$$
H\left(x_{n}, \nabla g\left(x_{n}\right)-A x_{n}\right) \leq H\left(y, \nabla g\left(x_{n}\right)-A x_{n}\right) .
$$

This implies that

$$
\begin{gathered}
-\left\langle x_{n}, \nabla g\left(x_{n}\right)-A x_{n}\right\rangle+g\left(x_{n}\right)+f\left(x_{n}\right) \\
\leq-\left\langle y, \nabla g\left(x_{n}\right)-A x_{n}\right\rangle+g(y)+f(y) .
\end{gathered}
$$

Since $\nabla g$ and $A$ are continuous and $f$ is lower semicontinuous, we conclude that

$$
\begin{aligned}
- & \langle x, \nabla g(x)-A x\rangle+g(x)+f(x) \\
& \leq-\langle y, \nabla g(x)-A x\rangle+g(y)+f(y) .
\end{aligned}
$$

Therefore,

$$
H(x, \nabla g(x)-A x) \leq H(y, \nabla g(x)-A x),
$$

which implies that $x \in V(y)$. Now, we prove that $V: C \rightarrow 2^{C}$ is a KKM mapping. Indeed, suppose $y_{1}, y_{2}, \ldots, y_{n} \in C$ and $0<a_{1}, a_{2}, \ldots, a_{n} \leq 1$ with $\sum_{i=1}^{n} a_{i}=1$. Let $z=\sum_{i=1}^{n} a_{i} y_{i}$. In view of the property (2) of $H$, we obtain

$$
\begin{aligned}
& H(z, \nabla g(z)-A z) \\
& =H\left(\sum_{i=1}^{n} a_{i} y_{i}, \nabla g(z)-A z\right) \leq \sum_{i=1}^{n} a_{i} H\left(y_{i}, \nabla g(z)-A z\right)
\end{aligned}
$$

and hence

$$
H(z, \nabla g(z)-A z) \leq \max _{1 \leq i \leq n} H\left(y_{i}, \nabla g(z)-A z\right) .
$$

Hence there exists at least one number $j=1,2, \ldots, n$, such that

$$
H(z, \nabla g(z)-A z) \leq H\left(y_{j}, \nabla g(z)-A z\right) .
$$

that is, $z \in V(y)$. Thus, $V$ is a KKM mapping.

If $x \in V\left(y_{0}\right)$, then $H(z, \nabla g(z)-A z) \leq H\left(y_{0}, \nabla g(z)-A z\right)$. By the definition of $H$, we obtain

$$
\begin{aligned}
- & \langle x, \nabla g(x)-A x\rangle+g(x)+f(x) \\
& \leq-\left\langle y_{0}, \nabla g(x)-A x\right\rangle+g\left(y_{0}\right)+f\left(y_{0}\right)
\end{aligned}
$$

which is equivalent to

$$
\left\langle y_{0}-x, \nabla g(x)-A x\right\rangle+g(x)+f(x) \leq g\left(y_{0}\right)+f\left(y_{0}\right) .
$$

Therefore,

$$
\begin{aligned}
V\left(y_{0}\right)=\{ & x \in C:\left\langle\nabla g(x)-A x, y_{0}-x\right\rangle \\
& \left.+g(x)+f(x) \leq g\left(y_{0}\right)+f\left(y_{0}\right)\right\} .
\end{aligned}
$$

In view of (49), we deduce that $V\left(y_{0}\right)$ is compact. It follows from Lemma 12 that $\bigcap_{y \in C} V(y) \neq \emptyset$. Hence there exists at least one $\left.x_{0} \in \bigcap_{y \in C} V(y)\right)$; that is,

$$
H\left(x_{0}, \nabla g\left(x_{0}\right)-A x_{0}\right) \leq H\left(y, \nabla g\left(x_{0}\right)-A x_{0}\right), \quad \forall y \in C .
$$

In view of the definition of Bregman $f$-projection operator $\operatorname{Proj}_{C}^{f, g}$, we conclude that

$$
x_{0} \in \operatorname{Proj}_{C}^{f, g}\left(\nabla g\left(x_{0}\right)-A x_{0}\right) .
$$

This completes the proof.

Theorem 14. Let $E$ be a reflexive Banach space and $g: E \rightarrow$ $\mathbb{R}$ a strongly coercive Bregman function which is bounded on bounded subsets and uniformly convex and uniformly smooth on bounded subsets of $E$. Let $f: E \rightarrow \mathbb{R} \cup\{+\infty\}$ be a proper, 
convex, lower semicontinuous function. Let $C$ be a nonempty, closed, and convex subset of $E$ and let $T: C \rightarrow C$ be a Bregman weak relatively nonexpansive mapping. Let $\left\{\alpha_{n}\right\}_{n \in \mathbb{N} \cup\{0\}}$ be a sequence in $(0,1)$ such that $\liminf _{n \rightarrow \infty} \alpha_{n}\left(1-\alpha_{n}\right)>0$. Let $\left\{x_{n}\right\}_{n \in \mathbb{N} \cup\{0\}}$ be a sequence generated by

$$
\begin{gathered}
x_{0}=x \in C \text { chosen arbitrarily, } \\
C_{0}=C, \\
y_{n}=\nabla g^{*}\left[\alpha_{n} \nabla g\left(x_{n}\right)+\left(1-\alpha_{n}\right) \nabla g\left(T x_{n}\right)\right], \\
C_{n+1}=\left\{z \in C_{n}: H\left(z, \nabla g\left(y_{n}\right)\right) \leq H\left(z, \nabla g\left(x_{n}\right)\right)\right\}, \\
x_{n+1}=\operatorname{Proj}_{C_{n+1}}^{g} x, n \in \mathbb{N} \cup\{0\},
\end{gathered}
$$

where $\nabla g$ is the gradient of $g$. Then $\left\{x_{n}\right\}_{n \in \mathbb{N}},\left\{T x_{n}\right\}_{n \in \mathbb{N}}$, and $\left\{y_{n}\right\}_{n \in \mathbb{N}}$ converge strongly to $\operatorname{Proj}_{F}^{g} x_{0}$.

Proof. We divide the proof into several steps.

Step 1. We prove that $C_{n}$ is closed and convex for each $n \in$ $\mathbb{N} \cup\{0\}$.

It is clear that $C_{0}=C$ is closed and convex. Let $C_{m}$ be closed and convex for some $m \in \mathbb{N}$. For $z \in C_{m}$, we see that

$$
H\left(z, \nabla g\left(y_{m}\right)\right) \leq H\left(z, \nabla g\left(x_{m}\right)\right)
$$

is equivalent to

$$
\begin{aligned}
&\left\langle z, \nabla g\left(x_{m}\right)-\nabla g\left(y_{m}\right)\right\rangle \\
& \leq g\left(y_{m}\right)-g\left(x_{m}\right) \\
& \quad+\left\langle x_{m}, \nabla g\left(x_{m}\right)\right\rangle-\left\langle y_{m}, \nabla g\left(y_{m}\right)\right\rangle .
\end{aligned}
$$

It could easily be seen that $C_{m+1}$ is closed and convex. Therefore, $C_{n}$ is closed and convex for each $n \in \mathbb{N} \cup\{0\}$.

Step 2. We claim that $F \subset C_{n}$ for all $n \in \mathbb{N} \cup\{0\}$.

It is obvious that $F \subset C_{0}=C$. Assume now that $F \subset C_{m}$ for some $m \in \mathbb{N}$. Employing Lemma 7, for any $w \in F \subset C_{m}$, we obtain

$$
\begin{aligned}
H( & \left.w, \nabla g\left(y_{m}\right)\right) \\
= & H\left(w, \nabla g\left(y_{m}\right)\right) \\
= & g(w)-\left\langle w, \nabla g\left(y_{m}\right)\right\rangle+g^{*}\left(\nabla g\left(y_{m}\right)\right)+f(w) \\
= & V\left(w, \alpha_{m} \nabla g\left(x_{m}\right)+\left(1-\alpha_{m}\right) \nabla g\left(T x_{m}\right)\right)+f(w) \\
= & g(w)-\left\langle w, \alpha_{m} \nabla g\left(x_{m}\right)+\left(1-\alpha_{m} \nabla g\left(T x_{m}\right)\right)\right\rangle \\
& +g^{*}\left(\alpha_{m} \nabla g\left(x_{m}\right)+\left(1-\alpha_{m}\right) \nabla g\left(T x_{m}\right)\right)+f(w) \\
\leq & \alpha_{m} g(w)+\left(1-\alpha_{m}\right) g(w) \\
& +\alpha_{m} g^{*}\left(\nabla g\left(x_{m}\right)\right)+\left(1-\alpha_{m}\right) g^{*}\left(\nabla g\left(T x_{m}\right)\right)+f(w) \\
= & \alpha_{m} V\left(w, \nabla g\left(x_{m}\right)\right)+\left(1-\alpha_{m}\right) V\left(w, \nabla g\left(T x_{m}\right)\right)+f(w)
\end{aligned}
$$

$$
\begin{aligned}
& =\alpha_{m} D_{g}\left(w, x_{m}\right)+\left(1-\alpha_{m}\right) D_{g}\left(w, T x_{m}\right)+f(w) \\
& \leq \alpha_{m} D_{g}\left(w, x_{m}\right)+\left(1-\alpha_{m}\right) D_{g}\left(w, x_{m}\right)+f(w) \\
& =D_{g}\left(w, x_{m}\right)+f(w) \\
& =V\left(w, \nabla g\left(x_{m}\right)\right)+f(w) \\
& =H\left(w, \nabla g\left(x_{m}\right)\right) .
\end{aligned}
$$

This proves that $w \in C_{m+1}$ and hence $F \subset C_{n}$ for all $n \in$ $\mathbb{N} \cup\{0\}$.

Step 3. We prove that $\left\{x_{n}\right\}_{n \in \mathbb{N}},\left\{y_{n}\right\}_{n \in \mathbb{N}}$, and $\left\{T x_{n}\right\}_{n \in \mathbb{N}}$ are bounded sequences in $C$.

Since $x_{n}=\operatorname{proj}_{C_{n}}^{g} x$, we get that

$$
H\left(x_{n}, \nabla g(x)\right) \leq H(w, \nabla g(x))
$$

for each $w \in F(T)$. This implies that the sequence $\left\{H\left(w, \nabla g\left(x_{n}\right)\right)\right\}_{n \in \mathbb{N}}$ is bounded and hence there exists $M_{1}>0$ such that

$$
H\left(x_{n}, \nabla g(x)\right) \leq M_{1}, \quad \forall n \in \mathbb{N} .
$$

We claim that the sequence $\left\{x_{n}\right\}_{n \in \mathbb{N}}$ is bounded. Assume on the contrary that $\left\|x_{n}\right\| \rightarrow \infty$ as $n \rightarrow \infty$. In view of Lemma 8 , there exist $x^{*} \in E^{*}$ and $a \in \mathbb{R}$ such that

$$
f(x) \geq\left\langle x_{n}, x^{*}\right\rangle+a, \quad \forall n \in \mathbb{N} .
$$

From the definition of Bregman distance, it follows that

$$
\begin{aligned}
M_{1} & \geq H\left(x_{n}, \nabla g(x)\right) \\
& =g\left(x_{n}\right)-g(x)-\left\langle x_{n}-x, \nabla g(x)\right\rangle+f\left(x_{n}\right) \\
& \geq g\left(x_{n}\right)-g(x)-\left\langle x_{n}, \nabla g(x)-x^{*}\right\rangle+\langle x, \nabla g(x)\rangle+a \\
\geq & g\left(x_{n}\right)-g(x)-\left\|x_{n}\right\|\left\|\nabla g(x)-x^{*}\right\| \\
& +\langle x, \nabla g(x)\rangle+a, \quad \forall n \in \mathbb{N} .
\end{aligned}
$$

Without loss of generality, we may assume that $\left\|x_{n}\right\| \neq 0$ for each $n \in \mathbb{N}$. This implies that

$$
\begin{aligned}
\frac{M_{1}}{\left\|x_{n}\right\|} \geq & \frac{g\left(x_{n}\right)}{\left\|x_{n}\right\|}-\frac{g(x)}{\left\|x_{n}\right\|}-\left\|\nabla g(x)-x^{*}\right\| \\
& +\frac{\langle x, \nabla g(x)\rangle}{\left\|x_{n}\right\|}+\frac{a}{\left\|x_{n}\right\|}, \quad \forall n \in \mathbb{N} .
\end{aligned}
$$

Since $g$ is strongly coercive, by letting $n \rightarrow \infty$ in (72), we conclude that $0 \geq \infty$, which is a contradiction. Therefore, $\left\{x_{n}\right\}_{n \in \mathbb{N}}$ is bounded. Since $\left\{T_{n}\right\}_{n \in \mathbb{N}}$ is an infinite family of Bregman weak relatively nonexpansive mappings from $C$ into itself, we have for any $q \in F$ that

$$
D_{g}\left(q, T x_{n}\right) \leq D_{g}\left(q, x_{n}\right), \quad \forall n \in \mathbb{N} \text {. }
$$


This, together with Definition 2 and the boundedness of $\left\{x_{n}\right\}_{n \in \mathbb{N}}$, implies that the sequence $\left\{T_{n} x_{n}\right\}_{n \in \mathbb{N}}$ is bounded.

Step 4. We show that $x_{n} \rightarrow v$ for some $v \in F$, where $v=$ $\operatorname{proj}_{F}^{g} x$.

From Step 3 we know that $\left\{x_{n}\right\}_{n \in \mathbb{N}}$ is bounded. By the construction of $C_{n}$, we conclude that $C_{m} \subset C_{n}$ and $x_{m}=$ proj $_{C_{m}}^{g} x \in C_{m} \subset C_{n}$ for any positive integer $m \geq n$. This, together with (23), implies that

$$
\begin{aligned}
D_{g} & \left(x_{m}, x_{n}\right) \\
= & D_{g}\left(x_{m}, \operatorname{proj}_{C_{n}}^{g} x\right) \leq D_{g}\left(x_{m}, x\right) \\
& \quad-D_{g}\left(\operatorname{proj}_{C_{n}}^{g} x, x\right)=D_{g}\left(x_{m}, x\right)-D_{g}\left(x_{n}, x\right) .
\end{aligned}
$$

In view of (21), we conclude that

$$
\begin{aligned}
D_{g}\left(x_{n}, x\right) & =D_{g}\left(\operatorname{proj}_{C_{n}}^{g} x, x\right) \leq D_{g}(w, x)-D_{g}\left(w, x_{n}\right) \\
& \leq D_{g}(w, x), \quad \forall w \in F \subset C_{n}, n \in \mathbb{N} \cup\{0\}
\end{aligned}
$$

It follows from (75) that the sequence $\left\{D_{g}\left(x_{n}, x\right)\right\}_{n \in \mathbb{N}}$ is bounded and hence there exists $M_{2}>0$ such that

$$
D_{g}\left(x_{n}, x\right) \leq M_{2}, \quad \forall n \in \mathbb{N} .
$$

In view of (64), we conclude that

$$
\begin{array}{r}
D_{g}\left(x_{n}, x\right) \leq D_{g}\left(x_{n}, x\right)+D_{g}\left(x_{m}, x_{n}\right) \leq D_{g}\left(x_{m}, x\right) \\
\forall m \geq n .
\end{array}
$$

This proves that $\left\{D_{g}\left(x_{n}, x\right)\right\}_{n \in \mathbb{N}}$ is an increasing sequence in $\mathbb{R}$

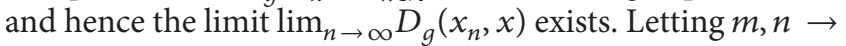
$\infty$ in (74), we deduce that $D_{g}\left(x_{m}, x_{n}\right) \rightarrow 0$. In view of Lemma 6, we obtain that $\left\|x_{m}-x_{n}\right\| \rightarrow 0$ as $m, n \rightarrow \infty$. This means that $\left\{x_{n}\right\}_{n \in \mathbb{N}}$ is a Cauchy sequence. Since $E$ is a Banach space and $C$ is closed and convex, we conclude that there exists $v \in C$ such that

$$
\lim _{n \rightarrow \infty}\left\|x_{n}-v\right\|=0
$$

Now, we show that $v \in F$. In view of Lemma 6 and (78), we obtain

$$
\lim _{n \rightarrow \infty} D_{g}\left(x_{n+1}, x_{n}\right)=0
$$

Since $x_{n+1} \in C_{n+1}$, we conclude that

$$
D_{g}\left(x_{n+1}, y_{n}\right) \leq D_{g}\left(x_{n+1}, x_{n}\right) \text {. }
$$

This, together with (79), implies that

$$
\lim _{n \rightarrow \infty} D_{g}\left(x_{n+1}, y_{n}\right)=0 \text {. }
$$

It follows from Lemma 6, (79), and (81) that

$$
\lim _{n \rightarrow \infty}\left\|x_{n+1}-x_{n}\right\|=0, \quad \lim _{n \rightarrow \infty}\left\|x_{n+1}-y_{n}\right\|=0 .
$$

In view of (78), we get

$$
\lim _{n \rightarrow \infty}\left\|y_{n}-u\right\|=0
$$

From (78) and (83), it follows that

$$
\lim _{n \rightarrow \infty}\left\|x_{n}-y_{n}\right\|=0
$$

Since $\nabla g$ is uniformly norm-to-norm continuous on any bounded subset of $E$, we obtain

$$
\lim _{n \rightarrow \infty}\left\|\nabla g\left(x_{n}\right)-\nabla g\left(y_{n}\right)\right\|=0 \text {. }
$$

Applying Lemma 6 we derive that

$$
\lim _{n \rightarrow \infty} D_{g}\left(y_{n}, x_{n}\right)=0 \text {. }
$$

It follows from the three-point identity (see (14)) that for any $w \in F$

$$
\begin{aligned}
& \left|D_{g}\left(w, x_{n}\right)-D_{g}\left(w, y_{n}\right)\right| \\
& =\mid D_{g}\left(w, y_{n}\right)+D_{g}\left(y_{n}, x_{n}\right) \\
& \quad \quad \quad\left\langle w-y_{n}, \nabla g\left(y_{n}\right)-\nabla g\left(x_{n}\right)\right\rangle-D_{g}\left(w, y_{n}\right) \mid \\
& =\left|D_{g}\left(y_{n}, x_{n}\right)-\left\langle w-y_{n}, \nabla g\left(y_{n}\right)-\nabla g\left(x_{n}\right)\right\rangle\right| \\
& \leq D_{g}\left(y_{n}, x_{n}\right)+\left\|w-y_{n}\right\|\left\|\nabla g\left(y_{n}\right)-\nabla g\left(x_{n}\right)\right\| \\
& \longrightarrow 0
\end{aligned}
$$

as $n \rightarrow \infty$.

The function $g$ is bounded on bounded subsets of $E$ and, thus, $\nabla g$ is also bounded on bounded subsets of $E^{*}$ (see, e.g., [22, Proposition 1.1.11], for more details). This implies that the sequences $\left\{\nabla g\left(x_{n}\right)\right\}_{n \in \mathbb{N}},\left\{\nabla g\left(y_{n}\right)\right\}_{n \in \mathbb{N}}$, and $\left\{\nabla g\left(T x_{n}\right): n \in \mathbb{N} U\right.$ $\{0\}\}$ are bounded in $E^{*}$.

In view of Proposition 4(3), we know that dom $g^{*}=E^{*}$ and $g^{*}$ is strongly coercive and uniformly convex on bounded subsets of $E^{*}$. Let $s_{1}=\sup \left\{\left\|\nabla g\left(x_{n}\right)\right\|,\left\|\nabla g\left(T x_{n}\right)\right\|: n \in \mathbb{N} \cup\right.$ $\{0\}\}$ and $\rho_{s_{1}}^{*}: E^{*} \rightarrow \mathbb{R}$ be the gauge of uniform convexity of the conjugate function $g^{*}$. We prove that for any $w \in F$

$$
\begin{aligned}
D_{g}\left(w, y_{n}\right) \leq & D_{g}\left(w, x_{n}\right)-\alpha_{n}\left(1-\alpha_{n}\right) \rho_{s_{1}}^{*} \\
& \times\left(\left\|\nabla g\left(x_{n}\right)-\nabla g\left(T x_{n}\right)\right\|\right) .
\end{aligned}
$$


Let us show (88). For any given $w \in F(T)$, in view of the definition of the Bregman distance (see (2)) and Lemma 6, we obtain

$$
\begin{aligned}
& D_{g}\left(w, y_{n}\right) \\
&=D_{g}\left(w, \nabla g^{*}\left[\alpha_{n} \nabla g\left(x_{n}\right)+\left(1-\alpha_{n}\right) \nabla g\left(T x_{n}\right)\right]\right) \\
&= V\left(w, \alpha_{n} \nabla g\left(x_{n}\right)+\left(1-\alpha_{n}\right) \nabla g\left(T x_{n}\right)\right) \\
&= g(w)-\left\langle w, \alpha_{n} \nabla g\left(x_{n}\right)+\left(1-\alpha_{n}\right) \nabla g\left(T x_{n}\right)\right\rangle \\
&+g^{*}\left(\alpha_{n} \nabla g\left(x_{n}\right)+\left(1-\alpha_{n}\right) \nabla g\left(T x_{n}\right)\right) \\
& \leq \alpha_{n} g(w)+\left(1-\alpha_{n}\right) g(w)-\alpha_{n}\left\langle w, \nabla g\left(x_{n}\right)\right\rangle \\
&-\left(1-\alpha_{n}\right)\left\langle w, \nabla g\left(T x_{n}\right)\right\rangle \\
&+\alpha_{n} g^{*}\left(\nabla g\left(x_{n}\right)\right)+\left(1-\alpha_{n}\right) g^{*}\left(\nabla g\left(T x_{n}\right)\right) \\
&-\alpha_{n}\left(1-\alpha_{n}\right) \rho_{s_{1}}^{*}\left(\left\|\nabla g\left(x_{n}\right)-\nabla g\left(T x_{n}\right)\right\|\right) \\
&= \alpha_{n} V\left(w, \nabla g\left(x_{n}\right)\right)+\left(1-\alpha_{n}\right) V\left(w, \nabla g\left(T x_{n}\right)\right) \\
&-\alpha_{n}\left(1-\alpha_{n}\right) \rho_{s_{1}}^{*}\left(\left\|\nabla g\left(x_{n}\right)-\nabla g\left(T_{n} x_{n}\right)\right\|\right) \\
&= \alpha_{n} D_{g}\left(w, x_{n}\right)+\left(1-\alpha_{n}\right) D_{g}\left(w, T x_{n}\right) \\
&-\alpha_{n}\left(1-\alpha_{n}\right) \rho_{s_{1}}^{*}\left(\left\|\nabla g\left(x_{n}\right)-\nabla g\left(T x_{n}\right)\right\|\right) \\
& \leq \alpha_{n} D_{g}\left(w, x_{n}\right)+\left(1-\alpha_{n}\right) D_{g}\left(w, x_{n}\right) \\
&-\alpha_{n}\left(1-\alpha_{n}\right) \rho_{s_{1}}^{*}\left(\left\|\nabla g\left(x_{n}\right)-\nabla g\left(T x_{n}\right)\right\|\right) \\
&= D_{g}\left(w, x_{n}\right)-\alpha_{n}\left(1-\alpha_{n}\right) \rho_{s_{1}}^{*}\left(\left\|\nabla g\left(x_{n}\right)-\nabla g\left(T x_{n}\right)\right\|\right) .
\end{aligned}
$$

In view of (87), we get that

$$
D_{g}\left(w, x_{n}\right)-D_{g}\left(w, y_{n}\right) \longrightarrow 0 \text { as } n \longrightarrow \infty .
$$

In view of (87) and (88), we conclude that

$$
\begin{gathered}
\alpha_{n}\left(1-\alpha_{n}\right) \rho_{s_{1}}^{*}\left(\left\|\nabla g\left(x_{n}\right)-\nabla g\left(T x_{n}\right)\right\|\right) \\
\leq D_{g}\left(w, x_{n}\right)-D_{g}\left(w, y_{n}\right) \longrightarrow 0
\end{gathered}
$$

as $n \rightarrow \infty$. From the assumption $\lim _{\inf _{n \rightarrow \infty}} \alpha_{n}\left(1-\alpha_{n}\right)>0$, we get

$$
\lim _{n \rightarrow \infty} \rho_{s_{1}}^{*}\left(\left\|\nabla g\left(x_{n}\right)-\nabla g\left(T x_{n}\right)\right\|\right)=0 .
$$

Therefore, from the property of $\rho_{s_{1}}^{*}$ we deduce that

$$
\lim _{n \rightarrow \infty}\left\|\nabla g\left(x_{n}\right)-\nabla g\left(T x_{n}\right)\right\|=0
$$

Since $\nabla g^{*}$ is uniformly norm-to-norm continuous on bounded subsets of $E^{*}$, we arrive at

$$
\lim _{n \rightarrow \infty}\left\|x_{n}-T x_{n}\right\|=0 .
$$

This implies that $v \in F(T)$.
Finally, we show that $v=\operatorname{proj}_{F}^{g} x$. From $x_{n}=\operatorname{proj}_{C_{n}}^{g} x$, we conclude that

$$
\left\langle z-x_{n}, \nabla g\left(x_{n}\right)-\nabla g(x)\right\rangle \geq 0, \quad \forall z \in C_{n} .
$$

Since $F \subset C_{n}$ for each $n \in \mathbb{N}$, we obtain

$$
\left\langle z-x_{n}, \nabla g\left(x_{n}\right)-\nabla g(x)\right\rangle \geq 0, \quad \forall z \in F .
$$

Letting $n \rightarrow \infty$ in (96), we deduce that

$$
\langle z-v, \nabla g(u)-\nabla g(x)\rangle \geq 0, \quad \forall z \in F .
$$

In view of (21), we have $v=\operatorname{proj}_{F}^{g} x$, which completes the proof.

Remark 15. Theorem 14 improves Theorem 4.1 of [20] in the following aspects.

(1) For the structure of Banach spaces, we extend the duality mapping to more general case, that is, a convex, continuous, and strongly coercive Bregman function which is bounded on bounded subsets and uniformly convex and uniformly smooth on bounded subsets.

(2) For the mappings, we extend the mapping from a relatively nonexpansive mapping to a Bregman weak relatively nonexpansive mapping. We remove the assumption $\widehat{F}(T)=F(T)$ on the mapping $T$ and extend the result to a Bregman weak relatively nonexpansive mapping, where $\widehat{F}(T)$ is the set of asymptotic fixed points of the mapping $T$.

(3) Theorems 9 and 10 extend and improve corresponding results of [20].

\section{Conflict of Interests}

The authors declare that there is no conflict of interests regarding the publishing of this paper.

\section{Acknowledgment}

This research was partially supported by a grant from NSC.

\section{References}

[1] D. van Dulst, "Equivalent norms and the fixed point property for nonexpansive mappings," The Journal of the London Mathematical Society, vol. 25, no. 1, pp. 139-144, 1982.

[2] K. Goebel and W. A. Kirk, Topics in Metric Fixed Point Theory, vol. 28, Cambridge University Press, Cambridge, UK, 1990.

[3] J.-P. Gossez and E. Lami Dozo, "Some geometric properties related to the fixed point theory for nonexpansive mappings," Pacific Journal of Mathematics, vol. 40, pp. 565-573, 1972.

[4] B. Halpern, "Fixed points of nonexpanding maps," Bulletin of the American Mathematical Society, vol. 73, pp. 957-961, 1967.

[5] Y.-Y. Huang, J.-C. Jeng, T.-Y. Kuo, and C.-C. Hong, "Fixed point and weak convergence theorems for point-dependent $\lambda$ hybrid mappings in Banach spaces," Fixed Point Theory and Applications, vol. 2011, article 105, 2011. 
[6] N. Hussain, E. Naraghirad, and A. Alotaibi, "Existence of common fixed points using Bregman nonexpansive retracts and Bregman functions in Banach spaces," Fixed Point Theory and Applications, vol. 2013, article 113, 2013.

[7] W. R. Mann, "Mean value methods in iteration," Proceedings of the American Mathematical Society, vol. 4, pp. 506-510, 1953.

[8] S. Reich, "Weak convergence theorems for nonexpansive mappings in Banach spaces," Journal of Mathematical Analysis and Applications, vol. 67, no. 2, pp. 274-276, 1979.

[9] S. Reich and S. Sabach, "Existence and approximation of fixed points of Bregman firmly nonexpansive mappings in reflexive Banach spaces," in Fixed-Point Algorithms for Inverse Problems in Science and Engineering, vol. 49, pp. 299-314, Springer, New York, NY, USA, 2010.

[10] H. F. Senter and W. G. Dotson, Jr., "Approximating fixed points of nonexpansive mappings," Proceedings of the American Mathematical Society, vol. 44, pp. 375-380, 1974.

[11] W. Takahashi, Nonlinear Functional Analysis, Fixed Point Theory and Its Applications, Yokohama Publishers, Yokohama, Japan, 2000.

[12] W. Takahashi and G.-E. Kim, "Approximating fixed points of nonexpansive mappings in Banach spaces," Mathematica Japonica, vol. 48, no. 1, pp. 1-9, 1998.

[13] W. Takahashi, Y. Takeuchi, and R. Kubota, "Strong convergence theorems by hybrid methods for families of nonexpansive mappings in Hilbert spaces," Journal of Mathematical Analysis and Applications, vol. 341, no. 1, pp. 276-286, 2008.

[14] W. Takahashi, N.-C. Wong, and J.-C. Yao, "Fixed point theorems and convergence theorems for generalized nonspreading mappings in Banach spaces," Journal of Fixed Point Theory and Applications, vol. 11, no. 1, pp. 159-183, 2012.

[15] S. Reich, "A weak convergence theorem for the alternating method with Bregman distances," in Theory and Applications of Nolinear Operators of Accretive and Monotone Type, vol. 178, pp. 313-318, Dekker, New York, NY, USA, 1996.

[16] G. Chen and M. Teboulle, "Convergence analysis of a proximallike minimization algorithm using Bregman functions," SIAM Journal on Optimization, vol. 3, no. 3, pp. 538-543, 1993.

[17] L. M. Bregman, "The relation method of finding the common point of convex sets and its application to the solution of problems in convex programming," USSR Computational Mathematics and Mathematical Physics, vol. 7, pp. 200-217, 1967.

[18] Y. Censor and A. Lent, "An iterative row-action method for interval convex programming," Journal of Optimization Theory and Applications, vol. 34, no. 3, pp. 321-353, 1981.

[19] E. Naraghirad and J.-C. Yao, "Bregman weak relatively nonexpansive mappings in Banach spaces," Fixed Point Theory and Applications, vol. 2013, article 141, 2013.

[20] K.-q. Wu and N.-j. Huang, "The generalised $f$-projection operator with an application," Bulletin of the Australian Mathematical Society, vol. 73, no. 2, pp. 307-317, 2006.

[21] X. Li, N.-J. Huang, and D. O’Regan, "Strong convergence theorems for relatively nonexpansive mappings in Banach spaces with applications," Computers \& Mathematics with Applications, vol. 60, no. 5, pp. 1322-1331, 2010.

[22] D. Butnariu and A. N. Iusem, Totally Convex Functions for Fixed Points Computation and Infinite Dimensional Optimization, vol. 40, Kluwer Academic Publishers, Dordrecht, The Netherlands, 2000.

[23] F. Kohsaka and W. Takahashi, "Proximal point algorithms with Bregman functions in Banach spaces," Journal of Nonlinear and Convex Analysis, vol. 6, no. 3, pp. 505-523, 2005.
[24] C. Zălinescu, Convex Analysis in General Vector Spaces, World Scientific Publishing, River Edge, NJ, USA, 2002.

[25] R. T. Rockafellar, "Characterization of the subdifferentials of convex functions," Pacific Journal of Mathematics, vol. 17, pp. 497-510, 1966.

[26] R. T. Rockafellar, "On the maximal monotonicity of subdifferential mappings," Pacific Journal of Mathematics, vol. 33, pp. 209216, 1970.

[27] E. Naraghirad, W. Takahashi, and J.-C. Yao, "Generalized retraction and fixed point theorems using Bregman functions in Banach spaces," Journal of Nonlinear and Convex Analysis, vol. 13, no. 1, pp. 141-156, 2012.

[28] D. Butnariu and E. Resmerita, "Bregman distances, totally convex functions, and a method for solving operator equations in Banach spaces," Abstract and Applied Analysis, vol. 2003, Article ID 84919, 39 pages, 2006.

[29] K. Deimling, Nonlinear Functional Analysis, Springer, Berlin, Germany, 1985.

[30] G. X.-Z. Yuan, KKM Theory and Applications in Nonlinear Analysis, Marcel Dekker, New York, NY, USA, 1990. 


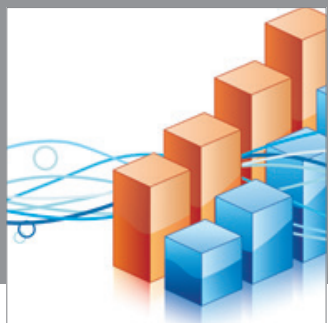

Advances in

Operations Research

mansans

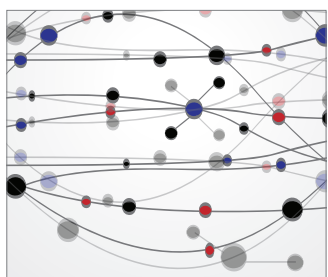

The Scientific World Journal
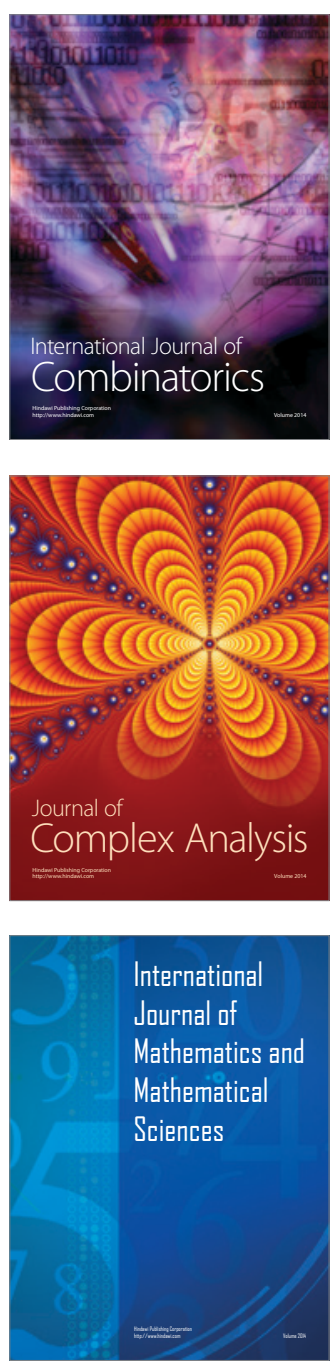
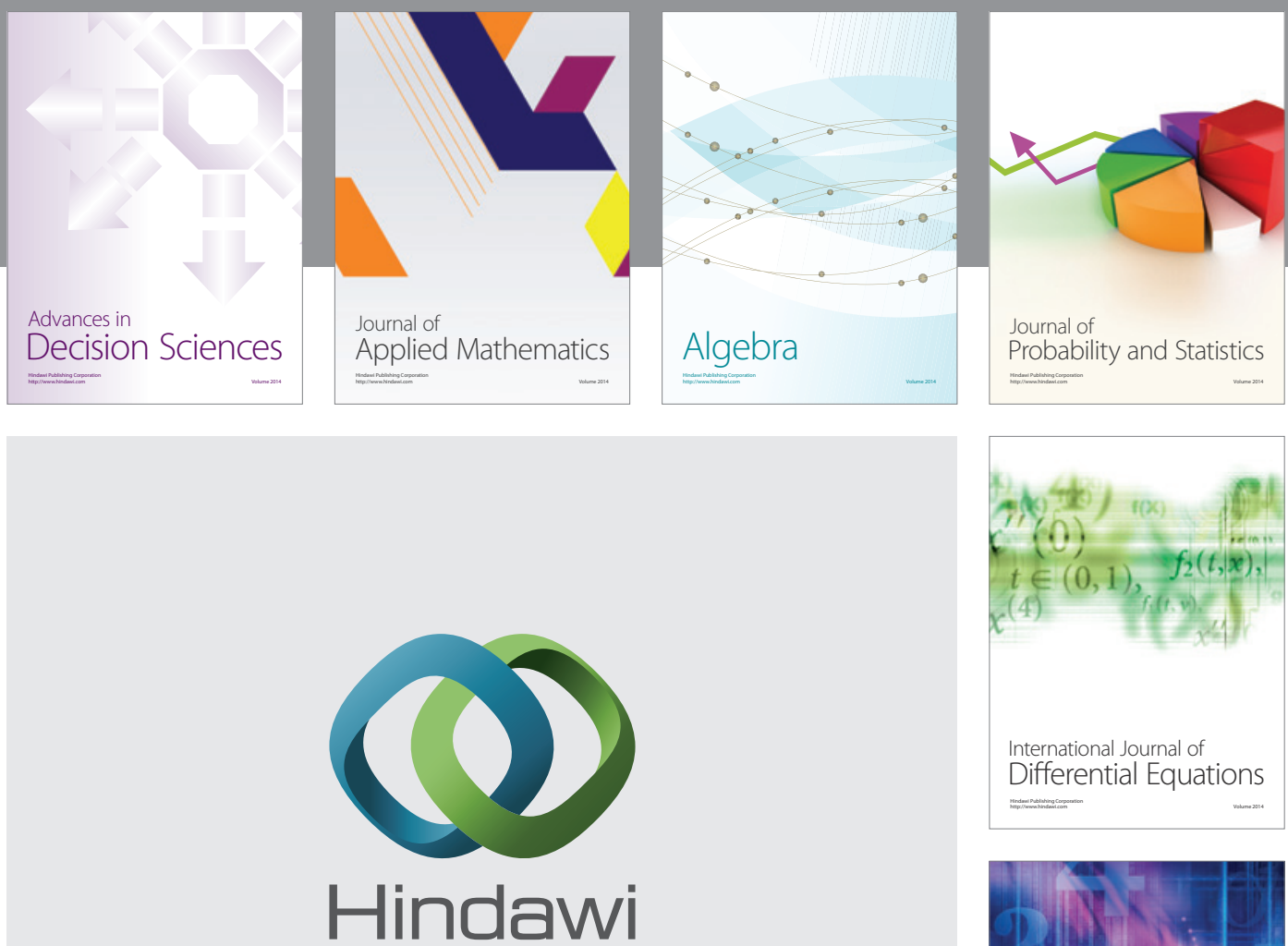

Submit your manuscripts at http://www.hindawi.com
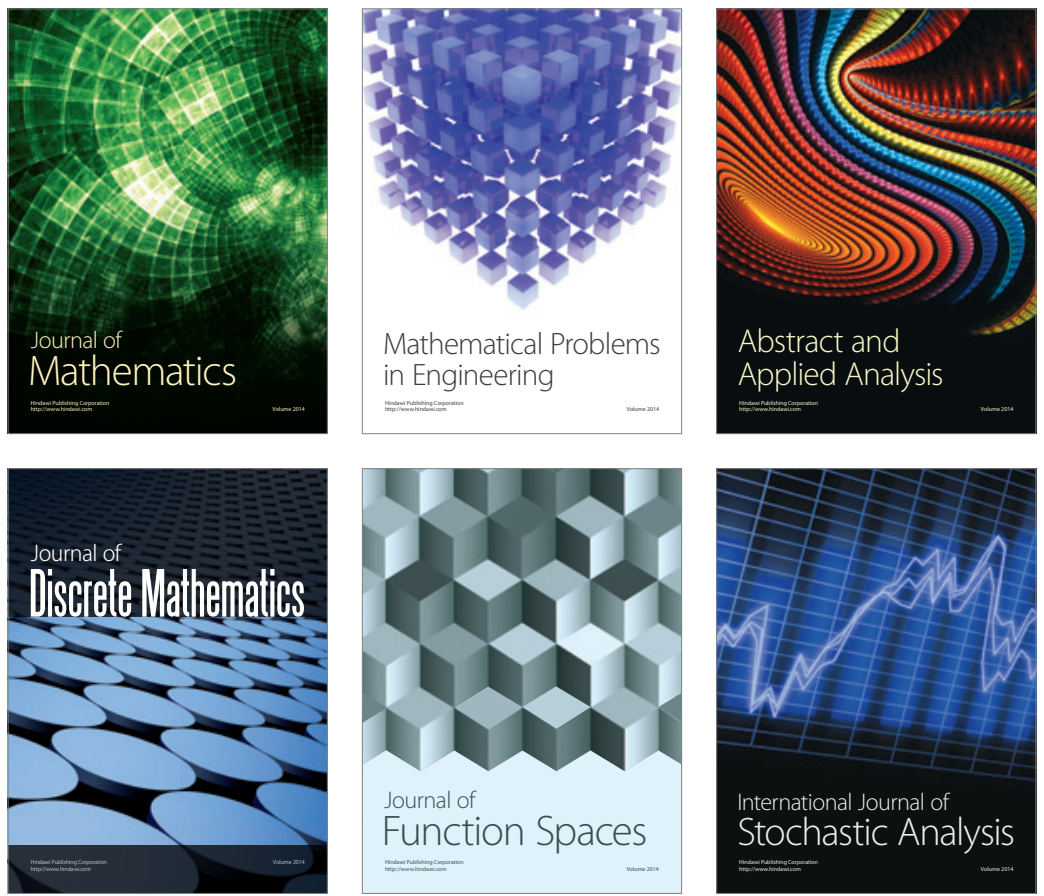

Journal of

Function Spaces

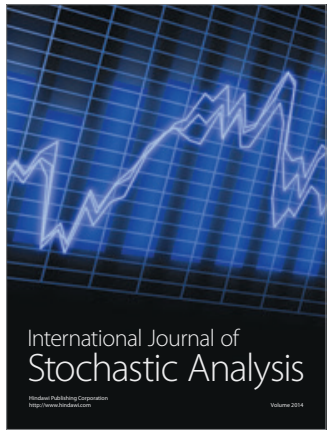

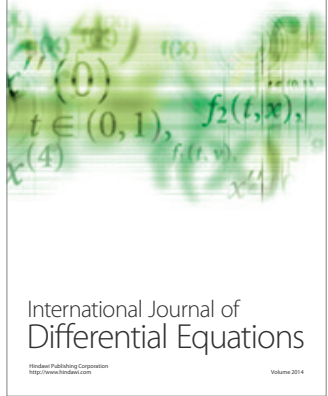
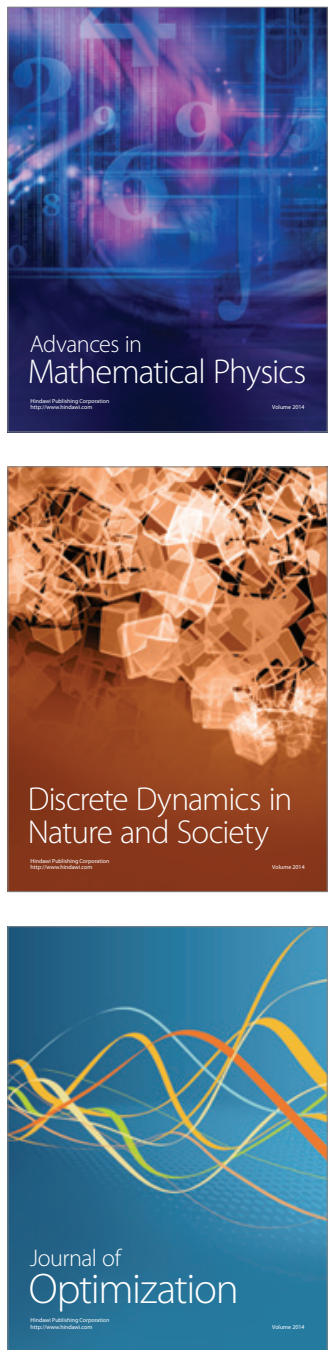